\title{
Apollonian Networks: Simultaneously Scale-Free, Small World, Euclidean, Space Filling, and with Matching Graphs
}

\author{
José S. Andrade, Jr., ${ }^{1}$ Hans J. Herrmann, ${ }^{1, *}$ Roberto F. S. Andrade, ${ }^{2}$ and Luciano R. da Silva ${ }^{3}$ \\ ${ }^{1}$ Departamento de Física, Universidade Federal do Ceará, 60451-970 Fortaleza, Ceará, Brazil \\ ${ }^{2}$ Instituto de Física, Universidade Federal da Bahia, 40210-340 Salvador, Bahia, Brazil \\ ${ }^{3}$ Departamento de Física, Universidade Federal do Rio Grande do Norte, 59072-970 Natal, Rio Grande do Norte, Brazil
}

(Received 12 June 2004; published 11 January 2005)

\begin{abstract}
We introduce a new family of networks, the Apollonian networks, that are simultaneously scale-free, small-world, Euclidean, space filling, and with matching graphs. These networks describe force chains in polydisperse granular packings and could also be applied to the geometry of fully fragmented porous media, hierarchical road systems, and area-covering electrical supply networks. Some of the properties of these networks, namely, the connectivity exponent, the clustering coefficient, and the shortest path are calculated and found to be particularly rich. The percolation, the electrical conduction, and the Ising models on such networks are also studied and found to be quite peculiar. Consequences for applications are also discussed.
\end{abstract}

DOI: 10.1103/PhysRevLett.94.018702

The study of networks has fruitfully inspired the understanding of transport and information flow within systems of many degrees of freedom during the last years. Many network types have been proposed and investigated serving to differentiate and elucidate effects ranging from clan formation, epidemics spreading, and logistic planning to earthquake prediction, neural activity, and immunological defenses [1-4]. Among a multitude of models, much attention has been dedicated to the study of scale-free networks, namely, networks that display a power-law degree distribution, $p(k) \propto k^{-\gamma}$, where $k$ is connectivity (degree) [1].

A network of crucial importance in the understanding of granular packings is the so called force network which connects the centers of mass of grains that have active, i.e., force transmitting, contacts. It is the key to understand the mechanical response of a soil or sand heap [5]. For simple monodisperse packings these networks are neither self-similar, nor scale-free, nor small-world. If particularly high densities are needed in order to get very high mechanical resistance, as it is, for instance, the case for high performance concrete (HPC) or ultrastrong ceramics rounded, polydisperse packings inspired in the famous Apollonian construction are used. We report in this Letter for the first time that, for the resulting packings, the force networks have many special properties including the ones mentioned above. This new class of networks, named Apollonian networks (ANs), can be either deterministic or random, are scale-free, display small-world effect, can be embedded in an Euclidean lattice, and show space filling as well as matching graph properties.

To define our network in its simplest deterministic version let us start with the problem of space-filling packing of spheres according to the ancient Greek mathematician Apollonius of Perga [6]. In its classical solution, three circles touch each other and the hole between them is filled
PACS numbers: 89.75.Hc, 64.60.Ak, 89.20.Hh, 89.75.Da

by the circle that touches all three, forming again three but much smaller holes that are then filled again each in the same way as shown in Fig. 1(a). The circle size distribution follows a power law with exponent of about 1.3 [6]. In fact, there exist many other topologies with different fractal dimensions [7]. This procedure can also be generalized to higher dimensions [8].

Apollonian tilings can describe dense granular packings and have also been used as toy models for turbulence and fragmentation. Connecting the centers of touching spheres by lines one obtains a network which in the classical case of Fig. 1(a) gives a triangulation that physically corresponds to the force network of the packing. We call the resulting network shown in Fig. 1(b) an "Apollonian network." Besides resembling the graphs introduced by Dodds [9] for the case of random packings, this network has also been used in the context of porous media [10].

First we show that ANs are scale-free. From Fig. 1(b) one can see that at each generation $n$ the number of sites $N$ increases by a factor 3 and the coordination of each site by a factor 2. More precisely, at generation $n(n=0,1,2, \ldots)$ there are $m(k, n)=3^{n}, 3^{n-1}, 3^{n-2} \ldots, 3^{2}, 3,1$, and 3 verti-
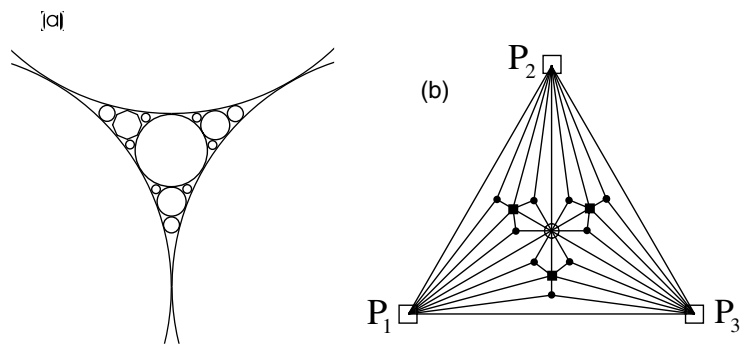

FIG. 1. (a) Classical Apollonian packing. (b) Apollonian network (AN); first, second, and third generation are symbols $\bigcirc, \mathbf{\square}$, and $\bullet$, respectively. 
ces with degree $k=3,3 \times 2,3 \times 2^{2}, \ldots, 3 \times 2^{n-1}, 3 \times$ $2^{n}$, and $2^{n+1}$, respectively, where the last number of vertices and degree correspond to the three corners, $P_{1}, P_{2}$, and $P_{3}$. Because of the discreteness of this degree spectrum, it is convenient to work with the cumulative distribution $P(k)=\sum_{k^{\prime} \geq k} m\left(k^{\prime}, n\right) / N_{n}$, where $N_{n}=3+\left(3^{n+1}-1\right) / 2$ is the number of sites at generation $n$. In order to compute the analog to our problem of the degree exponent $\gamma$, as commonly defined for continuum distributions [11], it is easy to show that $P(k) \propto k^{1-\gamma}$, with $\gamma=1+\ln 3 / \ln 2 \approx$ 2.585 .

Next, we show that ANs display small-world effect $[2,12,13]$. Accordingly, this means that the average length of the shortest path $l$ between two vertices as defined in Ref. [14] should grow slower than any positive power of the system size $N$. For the network in Fig. 1(b) we find that $l \propto(\ln N)^{\beta}$, with $\beta \approx 3 / 4$ as can be seen in Fig. 2. This is a novel intermediate behavior between small $(l \propto \ln N)$ and ultrasmall $(l \propto \ln \ln N)$ networks $[15,16]$.

The second property characterizing a small-world network is the clustering coefficient $C$ as defined in Ref. [14]. We find it to be 0.828 in the limit of large $N$, as shown in the inset of Fig. 2. This is a large value, comparable, for instance, with the cluster coefficient for the collaboration network of movie actors $C=0.79$ [14]. Consequently, since $L$ grows logarithmically and $C$ is close to unity, the AN indeed describes a small-world scenario [14].

As opposed to hierarchical lattices [11,17] used, for instance, in the Migdal-Kadanoff approximation, ANs are embedded in Euclidean space and can therefore describe geographical situations. In the case of Fig. 1(a) the sites form a multifractal point set in the asymptotic limit of infinitely many iterations [18]. In the ANs for $n \rightarrow \infty$, the bonds do not only completely cover the space (like a Peano curve), but also never cross each other. In this situation we have what is called a "matching graph" which is not the case for any other known scale-free network [1].

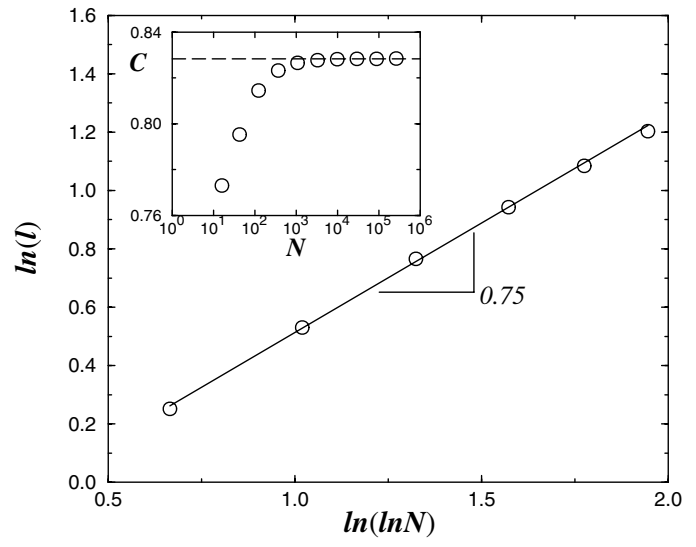

FIG. 2. Shortest path $l$ as a function of the number of sites $N$. The solid line is a guide to the eye with slope $3 / 4$. The inset shows the semilog plot of the clustering coefficient $C$ as a function of $N$. The dashed line is a guide to the eye for $C=$ 0.828 , observed at large values of $N$.
Now let us explore the properties of some well-known simple models defined on the lattice shown in Fig. 1(b). Thinking of an electrical supply system where the central site is the source (power station), the last generation sites are sinks (consumers), and all the connections have the same conductance, one can calculate the equivalent conductance $G$ of the network. Going from one generation to the next, the number of parallel system outputs increases by a factor 3 while at each node the number of outputs doubles. Because of the self-similarity, one has therefore doubled the conductance while increasing the number of sites by three. As a result, $G$ follows a power law, $G \propto N^{z}$, with $z=2 / 3$. As shown in Fig. 3, this exponent can be confirmed through computational simulation of the resistor network model. From Kirchhoff's law, the resulting system of linear algebraic equations are numerically solved using a sparse matrix inversion technique. This solution also allows us to compute the distribution of local currents $P(I)$, as shown in the inset of Fig. 3. Interestingly it follows a power law for large values of $I$ with an exponent -1.24 , as a consequence of the hierarchical choice of the output sites.

One can alternatively apply a potential drop between two corners of the system, i.e., $P_{1}$ and $P_{2}$ in Fig. 1(b). The resulting conductance numerically calculated as a function of the size of the network gives a power law, too, but with exponent 0.45 as shown in Fig. 3.

We now proceed with the study of bond percolation simultaneously applied among the three points $P_{1}, P_{2}$, and $P_{3}$ of Fig. 1(b) removing, however, the three direct connections between these points. Similarly to Ref. [19], we find that the percolation threshold $p_{c}$ goes to zero as the number of sites increases, according to a power law, as seen in Fig. 4. Assuming $p_{c} \propto \sqrt{N}^{-\frac{1}{\nu}}$, the slope yields an exponent $\nu \approx 3$.

Spin models can be defined on the AN in a variety of ways. We consider here that an Ising spin $s_{i}$ is placed on

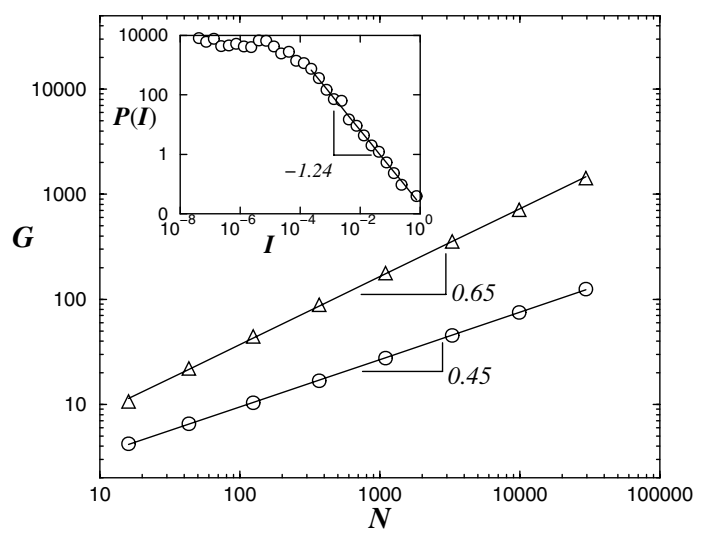

FIG. 3. Conductance $G$ as function of the number $N$ of sites when a potential is applied either between the center and all the sites of the last generation (upper curve) or between two corners of the network (lower curve). The inset shows the distribution of local currents for the first case. 


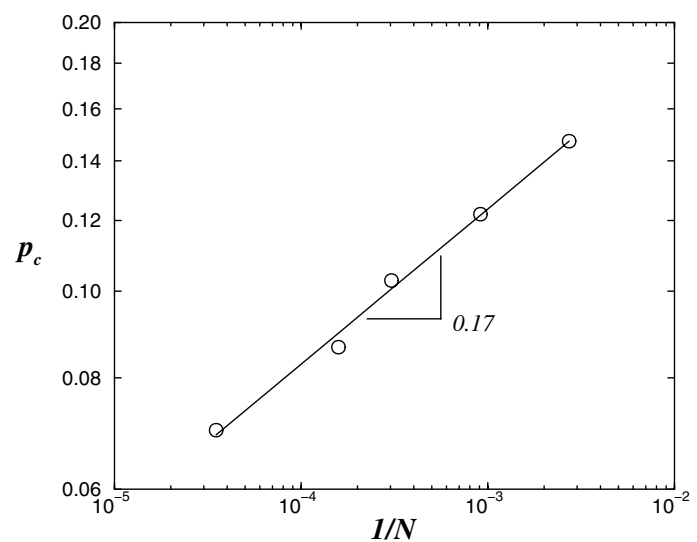

FIG. 4. Double logarithmic plot of the percolation threshold $p_{c}$ as function of the inverse system size $N$.

each site of the lattice. Between two sites we have a pair interaction constant $J_{n}$, where $n$ indicates that the link between the interacting spins is placed at the $n$th generation of the construction of the lattice. Moreover, the interactions are considered to be ferromagnetic $J_{n}>$ 0 , decaying with $n$ as $J_{n} \propto n^{-\alpha}$, and insensitive to the actual distance between the spins. With this model definition, it is straightforward to use a transfer matrix formalism to write down temperature dependent recurrence relations for the free energy $f_{n}$ and correlation length $\xi_{n}$ of the model at two subsequent generations, $f_{n+1}=$ $f_{n+1}\left(f_{n}, \xi_{n} ; T\right)$ and $\xi_{n+1}=\xi_{n+1}\left(f_{n}, \xi_{n} ; T\right) \quad[20,21]$. Exact values for these and any other functions in the thermodynamical limit $n \rightarrow \infty$ can be obtained through the numerical iteration of $f_{n+1}$ and $\xi_{n+1}$ and for their derivatives. The iteration process $n$ is stopped when the free energy per spin is the same in two subsequent generations within the first 16 digits.

The results obtained here are quite different from those for most self-similar lattices. The free energy, entropy, specific heat, and magnetization (respectively $f, s, c, m$ ) are found to be completely smooth for all values of $T$. A classical Schottky maximum dominates the behavior of $c$ while $s$ increases monotonically to $k_{B} \ln 2$. The presence of long-range order is observed as $m=1$ at low values of $T$. On the other hand, when $T$ is large, $m \rightarrow 0$ as $\exp (-T)$ for $\alpha=0$, with no noticeable presence of a critical temperature. For $\alpha>0$, we find a stronger decay, as $m \sim$ $\exp \left(-T^{\lambda}\right)$. The results for the correlation length are more subtle. $\xi$ diverges at low temperatures, expressing the evidence of long-range order. As $T$ increases beyond a given $T_{c}(n)$ it converges to a well defined value. However, if the iteration procedure is pursued to a value of $n$ larger than the one required for the convergence of $f_{n}$, we observe that $\xi$ keeps on diverging within a larger $T$ interval. This behavior therefore suggests the existence of an $n$ dependent effective critical temperature $T_{c}(n)$, as has been found by other authors for spin models on another scale-free lattice [22]. In Fig. 5 we show how $T_{c}(n)$ depends on $n$ for different values of $\alpha$. The power law

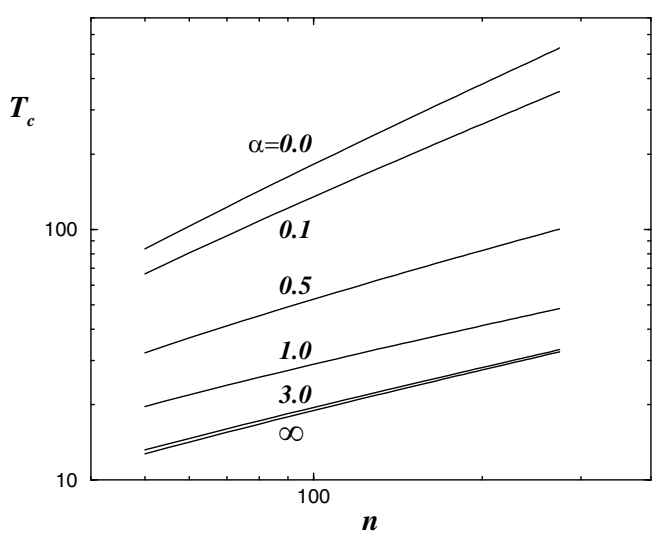

FIG. 5. Double logarithmic plot of the critical temperature as function of the generation number $n$ for different exponents $\alpha$.

$T_{c}(n) \sim n^{\tau(\alpha)}$, with $\tau$ going continuously from $\tau(0)=1$ to $\tau(\infty)=1 / 2$ indicates a novel kind of critical behavior.

It is important to mention that a more general version of the network shown in Fig. 1(b) is to choose at generation $n$ in each triangle a new site randomly and connect it to the three adjacent sites. In this sense the network can describe the porous fine structure inside a block of heterogeneous soil surrounded by larger pores or as the network of roads or supply lines going to smaller urban units surrounded by larger ones. There exists, in fact, a very large class of ANs obtained not only by changing the choice of the position of the sites inside the areas or changing the initial graph, but also having a different topology as classified in Ref. [23]. All these networks have the properties presented here, however, with different exponents if the topology is different.

ANs have the typical properties of the complex graphs in nature and society: large clustering coefficients, slower than logarithmic increase of the shortest path with number of sites, and a power-law degree distribution. In the context of critical phenomena, we did not find a finite critical point neither for percolation nor for the Ising model on ANs. The exponent $\nu=3$ controlling how the percolation threshold goes to zero with $N$ is unusually high while $T_{c}(n)$ of the Ising model diverges with a power- law in the number $n$ of generations.

An obvious and important application of ANs are the contact networks of space-filling packings. In other words, any configuration of rounded polydisperse grains which fills space (and that is realized, for example, in high performance concrete but up to a certain degree also in soils or sedimentary rocks) has a contact network which enters into the class we introduced as ANs. Considering this description of the force network in a polydisperse packing, the small-world nature of the lattice implies that between any two grains there are only a few contacts at best. If the medium is preconstrained, the electric conductance and elastic response have the same divergence [24], and we find that the system becomes more rigid with the inclusion of smaller particles following a power law (with 
exponent 0.45 in our topology) in the number of particles. Giving to each grain a remnant magnetization as discussed in Ref. [25], our result for the Ising model implies that, in the limit of many particles, one always finds a ferromagnetic response.

Interpreting our network as a porous medium, the fact that $p_{c}$ is equal to zero is compatible with Archie's law, namely, that there exists no finite critical porosity and the medium always percolates. If the network represents roads or electric supply lines between cities implies that they have small-world character and that the distribution of car flux or electrical currents follows a power law when going from larger to smaller sites. The results for the Ising model imply that opinions or cooperations can stabilize at any degree of interaction, and the percolation threshold being zero means that in the thermodynamic limit, the global connectivity cannot be disrupted, no matter how many connections are broken.

We have seen that the ANs introduced in this Letter can have several applications yielding reasonable conclusions while on the other hand, their critical properties are rather special. They should be studied in more detail including synchronization, spreading, path optimization, search algorithms, and more specific features of the real systems.

We thank CNPq, CAPES, FUNCAP, and the Max Planck prize for support. We also thank Jason Gallas and André Moreira for useful discussions.

*Also at Institute for Computer Physics, University of Stuttgart. Email: hans@ica1.uni-stuttgart.de.

[1] A.L. Barabasi, Linked: The New Science of Networks (Perseus Books Group, Cambridge MA, 2002).

[2] S. N. Dorogovtsev and J.F.F. Mendes, Evolution of Networks: From Biological Nets to the Internet and $W W W$ (Oxford University Press, New York, 2003).
[3] D. J. Watts, Small Worlds: The Dynamics of Networks Between Order and Randomness (Princeton University Press, Princeton, NJ, 1999).

[4] R. Pastor-Satorras and A. Vespignani, Evolution and Structure of the Internet: A Statistical Physics Approach, (Cambridge University Press, Cambridge, England, 2004).

[5] F. Radjai, D. Wolf, M. Jean, and J. J. Moreau, Phys. Rev. Lett. 80, 61 (1998).

[6] D. W. Boyd, Canadian Journal of Mathematics 25, 303 (1973).

[7] H. J. Herrmann, G. Mantica, and D. Bessis, Phys. Rev. Lett. 65, 3223 (1990).

[8] R. Mahmoodi Baram, H. J. Herrmann, and N. Rivier, Phys. Rev. Lett. 92, 044301 (2004).

[9] J. A. Dodds, J. Colloid Interface Sci. 77, 317 (1980).

[10] P. M. Adler, Int. J. Multiphase Flow 11, 91 (1985).

[11] S. N. Dorogovtsev, A. V. Goltsev, and J.F.F. Mendes, Phys. Rev. E 65, 066122 (2002).

[12] S. N. Dorogovtsev and J. F. F. Mendes, cond-mat/0404593.

[13] L. A. N. Amaral, A. Scala, M. Barthélemy, and H.E. Stanley, Proc. Nat. Acad. Sci. U.S.A. 97, 11149 (2000).

[14] D. J. Watts and S. H. Strogatz, Nature (London) 393, 440 (1998).

[15] A.F. Rozenfeld, R. Cohen, D. ben-Avraham, and S. Havlin, Phys. Rev. Lett. 89, 218701 (2002).

[16] R. Cohen and S. Havlin, Phys. Rev. Lett. 90, 058701 (2003).

[17] S. Jung, S. Kim, and B. Kahng, Phys. Rev. E 65, 056101 (2002).

[18] S. S. Manna and T. Vicsek, J. Stat. Phys. 64, 525 (1991).

[19] R. Cohen, K. Erez, D. ben-Avraham, and S. Havlin, Phys. Rev. Lett. 85, 4626 (2000); 86, 3682 (2001).

[20] M. Kohmoto, L. P. Kadanoff, and C. Tang, Phys. Rev. Lett. 50, 1870 (1983).

[21] R. F. S. Andrade, Phys. Rev. E 59, 150 (1999).

[22] A. Aleksiejuka, J. A. Holyst, and D. Stauffer, Physica A (Amsterdam) 310, 260 (2002).

[23] G. Oron and H. J. Herrmann, J. Phys. A 33, 1417 (2000).

[24] S. Alexander, J. Phys. (France) 45, 1939 (1984).

[25] S. Roux, A. Hansen, H. J. Herrmann, and J.-P. Vilotte, Geophys. Res. Lett. 20, 1499 (1993). 